\title{
Novel Bile Acids from Bear Bile Powder and Bile of Geese
}

\author{
Dan Bi, Xing-Yun Chai, Yue-Lin Song, Yu Lei, and Peng-Fei Tu* \\ State Key Laboratory of Natural and Biomimetic Drugs, School of Pharmaceutical Sciences, Peking University Health \\ Science Center; Beijing 100191, P. R. China. \\ Received December 21, 2008; accepted February 23, 2009; published online February 27, 2009
}

Two new bile acids, tauroselocholic acid (1) and tauroansocholic acid (2), a new natural bile acid, cygnocholic acid (3) were respectively isolated from bear bile powder Selenaretos thibetanus Cuvier and bile of geese Anser anser domesticus, together with seven known compounds. By spectrum analysis of MS, 1D and 2D NMR, the structures of the new compounds were elucidated as $3 \alpha, 7 \alpha, 9 \alpha$-trihydroxy-5 $\beta$-cholan-24-oic acid $N$-[2-sulfoethyl] amide (1), $3 \alpha, 5,7 \alpha$-trihydroxy-5 $\beta$-cholan-24-oic acid $N$-[2-sulfoethyl] amide (2) and $3 \alpha, 7 \alpha, 15 \alpha$-trihydroxy-5 $\beta$-cholan-24-oic acid (3).

Key words Selenaretos thibetanus; Anser anser domesticus; bile acid

Bile acids, as the water-soluble amphipathic end products of cholesterol metabolism, are important due to their roles in elimination of cholesterol and absorption of lipids and fatsoluble vitamins in the intestine. ${ }^{1)} \mathrm{C} 24$ bile acids found in most mammals are present in bile as $N$-acyl amidates (conjugates) of taurine or glycine. ${ }^{2)}$ All primary $\mathrm{C} 24$ bile acids have a hydroxyl group at C-3 which is from cholesterol and at C-7, as cholesterol $7 \alpha$-hydroxylation is the rate-limiting step in bile acid biosynthesis. Thus, chenodeoxycholic acid (CDCA; $3 \alpha, 7 \alpha$-dihydroxy-5 $\beta$-cholan-24-oic acid) is the root $\mathrm{C} 24$ bile acid. ${ }^{3)}$ Chenodeoxycholic acid and ursodeoxycholic acid (UDCA; $3 \alpha, 7 \alpha$-dihydroxy-5 $\beta$-cholanoic acid) are widely used for the treatment of cholesterol gall-stones and ursodeoxycholic acid has been introduced for the therapy of cholestatic liver diseases. ${ }^{4-9)}$ In the previous study, several bile acids including CDCA, CA, TCDCA, TUDCA, TCA were obtained from bile of Ursus arctos by Yamaguchi et $a l .{ }^{10)} \mathrm{In}$ order to search for new natural bioactive constituents from animal bile and provide chemical standards for the research on its quality control, two kinds of bile from different animals including Selenaretos thibetanus Cuvier and Anser anser domesticus were chemically investigated in our research group. This paper describes the isolation and structural determination of two novel bile acids, tauroselocholic acid (1), tauroansocholic acid (2), and a new natural bile acid, cygnocholic acid (3) which had been synthesized by Iida et al. ${ }^{11)}$ together with seven known compounds from these two kinds of bile.

\section{Results and Discussion}

Compound 1 was isolated as colorless syrup, and it showed positive Gregory Pascoe reaction, suggesting it to be a bile acid. Its molecular formula $\mathrm{C}_{26} \mathrm{H}_{45} \mathrm{NO}_{7} \mathrm{~S}$ was established by the pseudo-molecular ion peak at $\mathrm{m} / \mathrm{z} 514.2860$ $[\mathrm{M}-\mathrm{H}]^{-}$in the HR-ESI-MS and confirmed by the NMR data analysis. The IR spectrum indicated the presence of hydroxyl group $\left(3420 \mathrm{~cm}^{-1}\right)$, secondary amide group $(1647,1551$ $\left.\mathrm{cm}^{-1}\right)$ and sulfonic acid group $\left(1210 \mathrm{~cm}^{-1}\right)$.

The ${ }^{1} \mathrm{H}-\mathrm{NMR}$ data (Table 1) exhibited signals of two singlet methyl groups $\left[\delta_{\mathrm{H}} 0.69(3 \mathrm{H}, \mathrm{s}), 0.90(3 \mathrm{H}, \mathrm{s})\right]$, one doublet methyl group $\left[\delta_{\mathrm{H}} 0.95(3 \mathrm{H}, \mathrm{d}, J=6.5 \mathrm{~Hz})\right]$, two hydroxymethines $\left[\delta_{\mathrm{H}} 3.35,3.88(1 \mathrm{H}, \mathrm{m})\right]$, and an $\mathrm{AB}$ coupling system of a taurine group $\left[\delta_{\mathrm{H}} 2.95,3.55(2 \mathrm{H}, \mathrm{t}, J=7.0 \mathrm{~Hz})\right]$. Its ${ }^{13} \mathrm{C}$-NMR spectra (distortionless enhancement by polarization transfer (DEPT)) exhibited three methyls, twelve methylenes, seven methines and four quaternary carbons. Analysis of the ${ }^{13} \mathrm{C}$-NMR spectrum showed three hydroxylated carbons at $\delta$ $68.2(\mathrm{CH}), 73.1(\mathrm{CH})$ and $75.8(\mathrm{q} \mathrm{C})$, and one amide carbonyl at $\delta 176.6$. The above data showed high similarity with those of the known compound, $3 \alpha, 7 \alpha$-dihydroxy- $5 \beta$-cholanoic acid $N$-[2-sulfoethyl] amide $(\mathbf{8})^{10)}$ except for an additional hydroxyl group, which implied 1 to has $3 \alpha$-hydroxyl and $7 \alpha$ hydroxyl substituents. Interpretation of the ${ }^{1} \mathrm{H}-{ }^{1} \mathrm{H}$ correlation spectroscopy (COSY) spectrum led to the determination of partial structures I, II and III (Fig. 2). Partial structure I were connected to II by heteronuclear multiple bonding connectivity (HMBC) correlations (Fig. 2) from $\mathrm{H}_{3}-19$ to C-5, C-9, C10 , from $\mathrm{H}_{3}-18$ to $\mathrm{C}-12, \mathrm{C}-13, \mathrm{C}-14, \mathrm{C}-17$, and from $\mathrm{H}-11 \beta$ to $\mathrm{C}-8$. The structural moiety I was also connected to III by HMBC correlations (Fig. 2) from $\mathrm{H}_{2}-23$ and $\mathrm{H}_{2}-25$ to C-24. Analysis of ${ }^{13} \mathrm{C}-\mathrm{NMR}$ spectra through DEPT of $\mathbf{1}$ also showed that C-9 was a non-protonated carbon. Thus, the additional hydroxyl group was determined at $\mathrm{C}-9$, which was confirmed by $\mathrm{HMBC}$ correlations (Fig. 2) from $\mathrm{H}_{2}-1$ and $\mathrm{H}_{3}$ 19 to $\mathrm{C}-9(\delta 75.8)$.

The $\mathrm{H}_{3}-19 / \mathrm{H}-5 \beta$ correlation and the absence of the $\mathrm{H}_{3}$ $18 / \mathrm{H}-14 \alpha$ correlation in NOESY spectrum (Fig. 3) of compound 1 established cis type junction of rings-A/B and trans
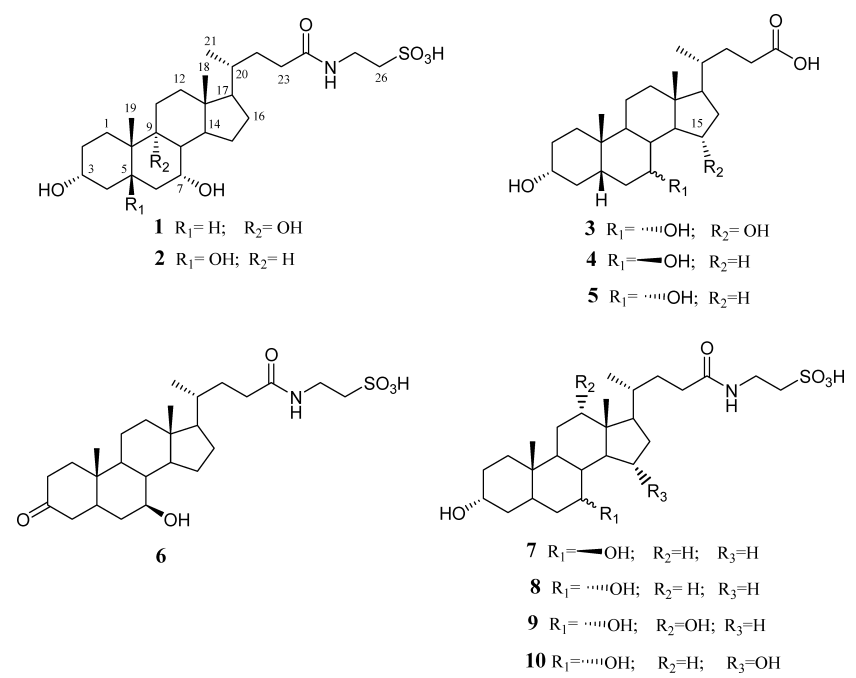

Fig. 1. Structures of Compounds $\mathbf{1}-\mathbf{1 0}$ 
Table 1. ${ }^{1} \mathrm{H}-\mathrm{NMR}$ Data (in $\mathrm{CD}_{3} \mathrm{OD}, 500 \mathrm{MHz}$ ) for $\mathbf{1}-\mathbf{3}, \delta$ in ppm

\begin{tabular}{|c|c|c|c|c|c|c|c|c|c|}
\hline \multirow{3}{*}{ Number } & \multicolumn{3}{|c|}{1} & \multicolumn{3}{|c|}{2} & \multicolumn{3}{|c|}{3} \\
\hline & \multirow{2}{*}{ Type } & \multicolumn{2}{|c|}{${ }^{1} \mathrm{H}$} & \multirow{2}{*}{ Type } & \multicolumn{2}{|c|}{${ }^{1} \mathrm{H}$} & \multirow{2}{*}{ Type } & \multicolumn{2}{|c|}{${ }^{1} \mathrm{H}$} \\
\hline & & $\alpha$ & $\beta$ & & $\alpha$ & $\beta$ & & $\alpha$ & $\beta$ \\
\hline 1 & $\mathrm{CH}_{2}$ & 1.62 & 1.68 & $\mathrm{CH}_{2}$ & 1.53 & 1.32 & $\mathrm{CH}_{2}$ & 1.83 & 1.01 \\
\hline 2 & $\mathrm{CH}_{2}^{2}$ & 1.20 & 1.55 & $\mathrm{CH}_{2}$ & 1.34 & 1.62 & $\mathrm{CH}_{2}^{2}$ & 1.35 & 1.63 \\
\hline 3 & $\mathrm{CH}^{2}$ & - & 3.34 & $\mathrm{CH}^{2}$ & - & 3.82 & $\mathrm{CH}^{2}$ & - & 3.39 \\
\hline 4 & $\mathrm{CH}_{2}$ & 1.25 & 1.85 & $\mathrm{CH}_{2}$ & 1.65 & 1.84 & $\mathrm{CH}_{2}$ & 2.16 & 1.63 \\
\hline 5 & $\mathrm{CH}$ & - & 1.35 & $\mathrm{C}^{2}$ & - & - & $\mathrm{CH}$ & - & 1.35 \\
\hline 6 & $\mathrm{CH}_{2}$ & 1.71 & 1.61 & $\mathrm{CH}_{2}$ & 2.52 & 1.67 & $\mathrm{CH}_{2}$ & 1.55 & 2.07 \\
\hline 7 & $\mathrm{CH}^{2}$ & - & 3.88 & $\mathrm{CH}^{2}$ & - & 3.82 & $\mathrm{CH}^{2}$ & - & 4.01 \\
\hline 8 & $\mathrm{CH}$ & - & 1.40 & $\mathrm{CH}$ & - & 1.48 & $\mathrm{CH}$ & - & 1.66 \\
\hline 9 & $\mathrm{C}$ & - & - & $\mathrm{CH}$ & 1.85 & - & $\mathrm{CH}$ & 1.86 & - \\
\hline 10 & $\mathrm{C}$ & - & - & $\mathrm{C}$ & - & - & $\mathrm{C}$ & - & - \\
\hline 11 & $\mathrm{CH}_{2}$ & 1.30 & 1.45 & $\mathrm{CH}_{2}$ & 1.48 & 1.36 & $\mathrm{CH}_{2}$ & 1.52 & 1.29 \\
\hline 12 & $\mathrm{CH}_{2}^{2}$ & 1.07 & 2.00 & $\mathrm{CH}_{2}^{2}$ & 1.16 & 2.00 & $\mathrm{CH}_{2}^{2}$ & 1.30 & 1.97 \\
\hline 13 & $\mathrm{C}^{2}$ & - & - & $\mathrm{C}^{2}$ & - & - & $\mathrm{C}$ & - & - \\
\hline 14 & $\mathrm{CH}$ & 1.17 & - & $\mathrm{CH}$ & 1.43 & - & $\mathrm{CH}$ & 1.52 & - \\
\hline 15 & $\mathrm{CH}_{2}$ & 1.35 & 1.83 & $\mathrm{CH}_{2}$ & 1.15 & 1.73 & $\mathrm{CH}$ & - & 3.97 \\
\hline 16 & $\mathrm{CH}_{2}^{2}$ & 1.55 & 1.45 & $\mathrm{CH}_{2}^{2}$ & 1.91 & 1.32 & $\mathrm{CH}_{2}$ & 1.78 & 1.88 \\
\hline 17 & $\mathrm{CH}^{2}$ & 1.10 & - & $\mathrm{CH}$ & 1.16 & - & $\mathrm{CH}$ & 1.46 & - \\
\hline 18 & $\mathrm{CH}_{3}$ & \multicolumn{2}{|c|}{0.69} & $\mathrm{CH}_{3}$ & \multicolumn{2}{|c|}{0.68} & $\mathrm{CH}_{3}$ & \multicolumn{2}{|c|}{0.73} \\
\hline 19 & $\mathrm{CH}_{3}$ & \multicolumn{2}{|c|}{0.90} & $\mathrm{CH}_{3}$ & \multicolumn{2}{|c|}{0.87} & $\mathrm{CH}_{3}$ & \multicolumn{2}{|c|}{0.92} \\
\hline 20 & $\mathrm{CH}$ & - & 1.38 & $\mathrm{CH}$ & - & 1.43 & $\mathrm{CH}$ & - & 1.44 \\
\hline 21 & $\mathrm{CH}_{3}$ & \multicolumn{2}{|c|}{0.95} & $\mathrm{CH}_{3}$ & \multicolumn{2}{|c|}{0.96} & $\mathrm{CH}_{3}$ & \multicolumn{2}{|c|}{0.94} \\
\hline 22 & $\mathrm{CH}_{2}$ & 1.75 & 1.25 & $\mathrm{CH}_{2}$ & 1.77 & 1.30 & $\mathrm{CH}_{2}$ & 1.78 & 1.32 \\
\hline 23 & $\mathrm{CH}_{2}^{2}$ & 2.18 & 2.05 & $\mathrm{CH}_{2}$ & 2.24 & 2.09 & $\mathrm{CH}_{2}$ & 2.33 & 2.21 \\
\hline 24 & $\mathrm{C}^{2}$ & - & - & $\mathrm{C}^{2}$ & - & - & $\mathrm{C}^{2}$ & - & - \\
\hline 25 & $\mathrm{CH}_{2}$ & \multicolumn{2}{|c|}{3.55} & $\mathrm{CH}_{2}$ & \multicolumn{2}{|c|}{3.57} & & & \\
\hline 26 & $\mathrm{CH}_{2}$ & \multicolumn{2}{|c|}{2.95} & $\mathrm{CH}_{2}$ & \multicolumn{2}{|c|}{2.95} & & & \\
\hline
\end{tabular}

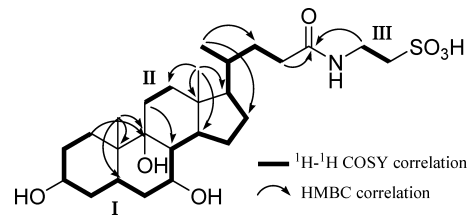

Fig. 2. $\quad{ }^{1} \mathrm{H}-{ }^{1} \mathrm{H}$ COSY and Key HMBC Correlations of $\mathbf{1}$

type junction of rings-C/D. The $\mathrm{H}_{3}-18 / \mathrm{H}-8 \beta$ and $\mathrm{H}_{3}-19 / \mathrm{H}-8 \beta$ correlations justified the trans type junction of rings- $\mathrm{B} / \mathrm{C}$, which also established the $\alpha$ arrangement of 9-OH. The observed NOESY correlations (Fig. 3) between $\mathrm{H}-3 / \mathrm{H}-1 \beta, \mathrm{H}-$ $3 / \mathrm{H}-4 \beta, \mathrm{H}_{3}-18 / \mathrm{H}-2 \beta, \mathrm{H}_{3}-18 / \mathrm{H}-11 \beta, \mathrm{H}_{3}-18 / \mathrm{H}-12 \beta, \mathrm{H}-6 \beta / \mathrm{H}-8$, $\mathrm{H}-14 / \mathrm{H}-15 \alpha, \mathrm{H}-14 / \mathrm{H}-16 \alpha, \mathrm{H}_{3}-21 / \mathrm{H}-22 \alpha$ and $\mathrm{H}_{3}-21 / \mathrm{H}-23 \alpha$ confirmed the relative configurations of 1 . The structure of compound 1 was thus determined as $3 \alpha, 7 \alpha, 9 \alpha$-trihydroxy$5 \beta$-cholan-24-oic acid $N$-[2-sulfoethyl] amide, and named tauroselocholic acid.

Compound 2, obtained as white amorphous powder, was an isomer of 1 by a pseudo-molecular ion peak $[\mathrm{M}-\mathrm{H}]^{-}$at $\mathrm{m} / \mathrm{z} 514.2844$ in the HR-ESI-MS spectrum. Its IR spectrum was very similar to that of $\mathbf{1}$. The ${ }^{1} \mathrm{H}-\mathrm{NMR}$ spectrum indicated the presence of three methyl groups at $\delta 0.68(3 \mathrm{H}, \mathrm{s})$, $0.87(3 \mathrm{H}, \mathrm{s})$ and $0.96(3 \mathrm{H}, \mathrm{d}, J=6.5)$, two hydroxymethines $\left[\delta_{\mathrm{H}} 3.82,3.82(1 \mathrm{H}, \mathrm{m})\right]$, and an $\mathrm{AB}$ coupling system of a taurine group $\left[\delta_{\mathrm{H}} 2.95,3.57(2 \mathrm{H}, \mathrm{t}, J=7.0 \mathrm{~Hz})\right]$. Its ${ }^{13} \mathrm{C}-\mathrm{NMR}$ spectra exhibited three methyls, twelve methylenes, seven methines and four quaternary carbons including three hydroxylated carbons at $\delta 68.6(\mathrm{CH}), 68.7(\mathrm{CH})$ and $76.1(\mathrm{q} \mathrm{C})$, and one amide carbonyl at $\delta$ 176.6. The above data were closely related to those of known compound methyl-

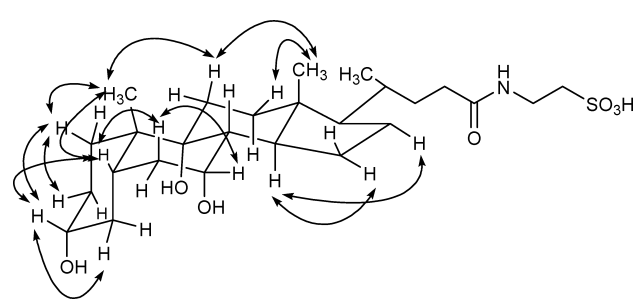

Fig. 3. Selected NOESY Correlations of $\mathbf{1}$

$3 \alpha, 5,7 \alpha$-trihydroxy-24-nor- $5 \beta$-chlan-23-oate, ${ }^{12)}$ except that signal of one methyl $\left(\delta_{\mathrm{H}} 3.67, \delta_{\mathrm{C}} 51.3\right)$ disappeared, and additional signals of a taurine group $\left(\delta_{\mathrm{H}} 2.95 ; \delta_{\mathrm{C}} 51.4\right.$ and $\left.\delta_{\mathrm{H}} 3.57 ; \delta_{\mathrm{C}} 36.6\right)$ and one methylene $\left(\delta_{\mathrm{H} \alpha} 1.77, \delta_{\mathrm{H} \beta} 1.30 ;\right.$ $\left.\delta_{\mathrm{C}} 33.2\right)$ appeared, suggesting 2 to be taurine conjugated $3 \alpha, 5 \beta, 7 \alpha$-trihydroxy $\mathrm{C} 24$ bile acid. A sequence of connectives through $\mathrm{H}-20 \beta, \mathrm{H}_{2}-22$ and $\mathrm{H}_{2}-23$, in turn, was observed in ${ }^{1} \mathrm{H}-{ }^{1} \mathrm{H}$ COSY, and the signal of $\mathrm{H}_{2}-25$ correlated $\mathrm{H}_{2}-26$. The connection C23-C24-C25 was supported by the HMBC correlations from $\mathrm{H}_{2}-23$ and $\mathrm{H}_{2}-25$ to $\mathrm{C}-24$. Therefore, the structure of 2 was identified as $3 \alpha, 5,7 \alpha$-trihydroxy- $5 \beta$ cholan-24-oic acid $N$-[2-sulfoethyl] amide and the trivial name tauroansocholic acid was given.

The molecular formula of $\mathbf{3}$ was determined as $\mathrm{C}_{24} \mathrm{H}_{40} \mathrm{O}_{5}$ by HR-ESI-MS at $m / z 431.2751[\mathrm{M}+\mathrm{Na}]^{+}$. The IR spectrum indicated the presence of the hydroxyl group $\left(3402 \mathrm{~cm}^{-1}\right)$. The NMR data (Tables 1,2$)$ were in good agreement with those of the known compound $3 \alpha, 7 \alpha, 15 \alpha$-trihydroxy- $5 \beta$ cholan-24-oyl taurine (10) ${ }^{13)}$ except for signals due to absence of taurine group at the side chain. Compound $\mathbf{3}$ was further determined as $3 \alpha, 7 \alpha, 15 \alpha$-trihydroxy- $5 \beta$-cholan-24- 
Table 2. ${ }^{13} \mathrm{C}-\mathrm{NMR}$ Data (in $\mathrm{CD}_{3} \mathrm{OD}, 125 \mathrm{MHz}$ ) for $\mathbf{1}-\mathbf{3}, \boldsymbol{\delta}$ in ppm

\begin{tabular}{|c|c|c|c|}
\hline Number & 1 & 2 & 3 \\
\hline 1 & 47.3 & 31.1 & 36.5 \\
\hline 2 & 30.7 & 30.9 & 31.2 \\
\hline 3 & 73.1 & 68.6 & 72.7 \\
\hline 4 & 29.7 & 44.9 & 40.0 \\
\hline 5 & 42.8 & 76.1 & 42.9 \\
\hline 6 & 43.7 & 45.9 & 34.1 \\
\hline 7 & 68.2 & 68.7 & 69.0 \\
\hline 8 & 43.8 & 40.1 & 40.5 \\
\hline 9 & 75.8 & 36.7 & 35.4 \\
\hline 10 & 40.2 & 41.2 & 35.9 \\
\hline 11 & 22.4 & 21.9 & 21.8 \\
\hline 12 & 41.4 & 40.8 & 41.6 \\
\hline 13 & 44.6 & 43.5 & 44.9 \\
\hline 14 & 57.6 & 51.4 & 59.6 \\
\hline 15 & 27.9 & 24.7 & 72.9 \\
\hline 16 & 30.7 & 29.2 & 41.1 \\
\hline 17 & 56.4 & 57.2 & 55.6 \\
\hline 18 & 12.6 & 12.1 & 13.5 \\
\hline 19 & 17.0 & 16.6 & 23.4 \\
\hline 20 & 36.6 & 36.9 & 36.2 \\
\hline 21 & 19.0 & 18.9 & 18.5 \\
\hline 22 & 33.2 & 33.2 & 32.2 \\
\hline 23 & 34.2 & 34.2 & 32.0 \\
\hline 24 & 176.6 & 176.6 & 178.2 \\
\hline 25 & 36.8 & 36.6 & - \\
\hline 26 & 51.5 & 51.4 & - \\
\hline
\end{tabular}

oic acid by interpretation of 2D NMR spectrum, and had been named as cygnocholic acid by Kakiyama et al. ${ }^{13)}$

Seven known compounds among six compounds from bear bile powder, ursodeoxycholic acid (4), ${ }^{14}$ chenodeoxycholic acid (5), ${ }^{10)}$ 2-[[(5 $\left.\beta, 7 \alpha\right)$-7-hydroxy-3,24-dioxocholan24-yl]amino]-ethanesulfonic acid (6), ${ }^{15)}$ tauroursodeoxycholic acid (7), ${ }^{10)}$ taurochenodeoxycholic acid (8), ${ }^{10)}$ taurocholic acid (9), ${ }^{10)}$ and $3 \alpha, 7 \alpha, 15 \alpha$-trihydroxy-5 $\beta$-cholan-24oyl taurine $(\mathbf{1 0})^{13)}$ from geese bile, were identified by comparing their NMR data with those reported.

\section{Experimental}

General Procedures Optical rotations were measured on a PerkinElmer-243B digital polarimeter. IR spectra were measured on a NEXUS-470 FTIR (Nicolet) spectrometer, $\mathrm{KBr}$ pellets, in $\mathrm{cm}^{-1}$. NMR spectra were recorded on a VARIAN INOVA 500 NMR spectrometer with chemical shifts shown as $\delta$-values (ppm) and TMS as an internal standard. ESI-MS were measured on a QSTAR (ABI, U.S.A.) mass spectrometer and HR-ESI-MS on a Bruker APEX II FT-ICR-MS mass spectrometer. Semi-preparative HPLC was carried out using a Waters 600 Pump with 600 controller (Waters C18 Nova-Pak column, $300 \times 7.8 \mathrm{~mm}, 5 \mu \mathrm{m}$ ), with ELSD detector (Alltech). All solvents used were of analytical grade (Beijing Chemical and Industry Factory). Silica gel (Qingdao Mar. Chem. Ind. Co., Ltd.), Sephadex LH-20 gel (Pharmacia) and $\mathrm{C}_{18}$ reverse-phased silica gel (150-200 mesh, Merck, performed by applying a $\mathrm{N}_{2}$ pressure of $0.12 \mathrm{MPa}$ ) were used for column chromatography.

Biological Materials Bear bile powder was prepared by drying the bile drained from live wild bears Selenaretos thibetanus CUvIER, artificially bred and raised in Hei Bao Medicine Group in China. The gallbladders from geese Anser anser domesticus were provided by He Wang Breeding Center of Landaise geese, Beijing, China.

Extraction and Isolation Bear bile powder $(190 \mathrm{~g})$ was dissolved in water and successively partitioned with EtOAc and $n-\mathrm{BuOH}$. The EtOAC extract $(8 \mathrm{~g})$ was subjected to silica gel chromatography column (CC) and eluted with $\mathrm{PE}-\mathrm{Me}_{2} \mathrm{CO}(20: 1-1: 5)$ to yield four fractions (Frs. 1-4). Fr. 3 was further subjected to silica gel $\mathrm{CC}$ and eluted with $\mathrm{CHCl}_{3}-\mathrm{EtOAC}$ $(3: 2)$ to obtain $4(10.0 \mathrm{mg})$ and $5(6.0 \mathrm{mg})$. The $n-\mathrm{BuOH}$ extract $(170 \mathrm{~g})$ was subjected to silica gel $\mathrm{CC}$ and eluted with EtOAc- $\mathrm{MeOH}-\mathrm{H}_{2} \mathrm{O}(8: 2: 0.5)$ to yield Frs. $1-6$. Fr. 3 was further subjected to silica gel $\mathrm{CC}$ and eluted with $\mathrm{CHCl}_{3}-\mathrm{MeOH}(4: 1)$ followed by purification on Sephadex $\mathrm{LH}-20\left(\mathrm{CHCl}_{3}-\right.$ $\mathrm{MeOH}, 1: 1)$ to afford $6(10.0 \mathrm{mg})$. Fr. 5 was subjected to silica gel vacuum liquid chromatography and eluted with $\mathrm{CHCl}_{3}-\mathrm{MeOH}(10: 1)$ to obtain Frs. 5-I-IV. Fr. 5-III further using ODS $\left(\mathrm{MeOH}-\mathrm{H}_{2} \mathrm{O}, 6: 4\right)$ afforded $7(224 \mathrm{mg})$ and $8(354 \mathrm{mg})$. Fr. 5-IV was re-subjected to ODS $\left(\mathrm{MeOH}-\mathrm{H}_{2} \mathrm{O}, 4: 6\right)$ to yield $1(7.0 \mathrm{mg})$ and $\mathbf{9}(90 \mathrm{mg})$.

The gallbladders from geese were cut and the bile (5.01) was partitioned with EtOAc and $n$-BuOH. The $n$ - $\mathrm{BuOH}$ extract $(200 \mathrm{~g})$ was subjected to silica gel column and eluted with $\mathrm{CHCl}_{3}-\mathrm{MeOH}(5: 1-2: 1)$ to yield Frs. $1-$ 3. Further isolation of Fr. 3 by ODS $\left(\mathrm{MeOH}-\mathrm{H}_{2} \mathrm{O}, 4: 6\right)$ provided Frs. 3-I and II. Further purification of Fr. 3-I by prep. HPLC $\left(\mathrm{MeOH} / \mathrm{H}_{2} \mathrm{O}(0.5 \%\right.$ $\left.\left.\mathrm{CF}_{3} \mathrm{COOH}\right), 65: 35\right)$ gave $2(6 \mathrm{mg})$. Fr. 3-II was subjected to silica gel column and eluted with EtOAc- $\mathrm{MeOH}-\mathrm{H}_{2} \mathrm{O}(8: 2: 0.5)$ to yield $10(40 \mathrm{mg})$. The EtOAc extract $(30 \mathrm{~g})$ was subjected to silica gel column and eluted with PE- $\mathrm{Me}_{2} \mathrm{CO}(20: 1-1: 5)$ to yield Frs. $1-7$. Fr. 5 (600 mg) was applied to silica gel CC with $\mathrm{CHCl}_{3}-$ EtOAc $(1: 3)$ elution to afford $3(10.0 \mathrm{mg})$.

Compound 1: Colorless syrup; $[\alpha]_{\mathrm{D}}^{20}+21.3^{\circ}(c=0.16, \mathrm{MeOH}) ; \mathrm{IR}(\mathrm{KBr})$ $v_{\max } \mathrm{cm}^{-1}: 3420,2931,2869,1647,1551,1453,1210,1047 ;{ }^{1} \mathrm{H}-\mathrm{NMR}$ $\left(\mathrm{CD}_{3} \mathrm{OD}, 500 \mathrm{MHz}\right)$, see Table $1 ;{ }^{13} \mathrm{C}-\mathrm{NMR}\left(\mathrm{CD}_{3} \mathrm{OD}, 125 \mathrm{MHz}\right)$, see Table 2. HR-ESI-MS $m / z$ 514.2860 [M-H] $]^{-}$(Calcd for $\mathrm{C}_{26} \mathrm{H}_{44} \mathrm{NO}_{7} \mathrm{~S}$ : 514.2844).

Compound 2: Colorless syrup; $[\alpha]_{\mathrm{D}}^{20}+14.8^{\circ}(c=0.14, \mathrm{MeOH})$. IR (KBr) $v_{\max } \mathrm{cm}^{-1}: 3425,2928,2859,1732,1635,1160,1037 .{ }^{1} \mathrm{H}-\mathrm{NMR}\left(\mathrm{CD}_{3} \mathrm{OD}\right.$, $500 \mathrm{MHz})$, see Table $1 ;{ }^{13} \mathrm{C}-\mathrm{NMR}\left(\mathrm{CD}_{3} \mathrm{OD}, 125 \mathrm{MHz}\right)$, see Table 2. HR-ESIMS $m / z$ 514.2857 [M-H] $]^{-}$(Calcd for $\left.\mathrm{C}_{26} \mathrm{H}_{44} \mathrm{NO}_{7} \mathrm{~S}: 514.2844\right)$.

Compound 3: Colorless syrup; $[\alpha]_{\mathrm{D}}^{20}+7.6^{\circ}(c=0.08, \mathrm{MeOH})$. IR (KBr) $v_{\max } \mathrm{cm}^{-1}: 3402,2931,2869,1731,1452,1371,1080,983 \mathrm{~cm}^{-1}$. ${ }^{1} \mathrm{H}-\mathrm{NMR}$ $\left(\mathrm{CD}_{3} \mathrm{OD}, 500 \mathrm{MHz}\right)$, see Table $1 ;{ }^{13} \mathrm{C}-\mathrm{NMR}\left(\mathrm{CD}_{3} \mathrm{OD}, 125 \mathrm{MHz}\right)$, see Table 2. HR-ESI-MS $m / z 431.2751[\mathrm{M}+\mathrm{Na}]^{+}$(Calcd for $\mathrm{C}_{24} \mathrm{H}_{40} \mathrm{O}_{5} \mathrm{Na}$ : 431.2768).

Present chemical investigation has resulted in the isolation and characterization of two new bile acids and a new natural bile acid together with seven known compounds. It will be worthwhile to investigate the biological potential of these molecules. Moreover, in contrast to most small molecules found in vertebrates, bile acids are strikingly diverse in their pattern of hydroxylation and side chain structure. ${ }^{16)}$ Bile acid structure shows a pattern of progressive molecular development in the course of vertebrate evolution. ${ }^{17)}$ The sites of an additional hydroxylation on the cholanoic acid nucleus in primary bile acids have been identified at $6 \alpha, 6 \beta, 12 \alpha, 1 \alpha, 1 \beta, 15 \alpha$ and $16 \alpha .{ }^{13)}$ With this report, two new additional sites of hydroxylation on the cholanoic acid nucleus in primary bile acids were discovered at C-9 and C-5. These bile acid structures may provide the useful information for phylogenesis.

\section{References}

1) Hofmann A. F., "The Liver: Biology and Pathobiology,” ed. by Arias I. M., Boyer J. L., Fausto N., Jakoby W. B., Schachter D. A., Raven Press, New York, 1994, pp. 677-718.

2) Moschetta A., Xu F., Hagey L. R., van Berge-Henegouwen G. P., van Erpecum K. J., Brouwers J. F., Cohen J. C., Bierman M., Hobbs H. H., Steinbach J. H., Hofmann A. F., J. Lipid Res., 46, 2221-2232 (2005).

3) Kakiyama G., Tamegai H., Iida T., Mitamura K., Ikegawa S., Goto T., Mano N., Goto J., Holz P., Hagey L. R., Hofmann A. F., J. Lipid Res., 48, 2682-2692 (2007).

4) Ward A., Brogden R. N., Heel R. C., Speight T. M., Avery G. S., Drugs, 27, 95-131 (1984).

5) Stiehl A., Raedach R., Czygan P., Goetz R., Maenner C., Walker S., Kommerell B., Gastroenterology, 79, 1192-1196 (1970).

6) Roda E., Bazzoli F., Labate A. M., Mazzella G., Roda A., Sama C., Festi D., Aldini R., Taroni F., Barbara L., Hepatology, 2, 804-810 (1982).

7) Leuschner U., Fischer H., Kurtz W., Giildutuna S., Hiibner K., Hellstern A., Gatzen M., Leuschner M., Gastroenterology, 96, 1268-1274 (1989).

8) Poupon R., Chretien Y., Poupon R. E., Ballet E., Calmus Y., Darnis F., Lancet, 1, 834-846 (1987).

9) Hofmann A. F., Popper H., Lancet, 2, 398-399 (1987).

10) Yamaguchi S., Qian Z. Z., Nohara T., Chem. Pharm. Bull., 46, 16531655 (1998).

11) Iida T., Hikosaka M., Kakiyama G., Shiraishi K., Schteingart C. D., Hagey L. R., Ton-Nu H. T., Hofmann A. F., Mano N., Goto J., Nambara T., Chem. Pharm. Bull., 50, 1327-1334 (2002).

12) Schteingart C. D., Hagey L. R., Setchell K. D. R., Hofmann A. F., J. Biol. Chem., 268, 11239-11246 (1993).

13) Kakiyama G., Iida T., Goto T., Mano N., Goto J., Nambara T., Hagey 
L. R., Schteingart C. D., Hofmann A. F., J. Lipid Res., 47, 1551-1558 (2006).

14) Waterhous D. V., Barnes S., Muccio D. D., J. Lipid Res., 26, 10681078 (1985).

15) Zhang X., Wang D. Q., Liu M. L., Chin. J. Mag. Reson., 16, 33-39 (1999).
16) Hofmann A. F., Schteingart C. D., Hagey L. R., "Bile Acids and Liver Diseases (International Falk Workshop)," ed. by Paumgartner G., Beuers U., Kluwer Academic Publishers, Boston, 1995, pp. 3-30.

17) Hagey L. R., "Bile Acid Biodiversity in Vertebrates: Chemistry and Evolutionary Implication," Ph.D. Dissertation, University of California, CA, 1992. 